\title{
Efeitos de antimicrobianos, prebióticos, probióticos e extratos vegetais sobre a microbiota intestinal, a freqüência de diarréia e o desempenho de leitões recém-desmamados ${ }^{1}$
}

\section{Carlos Eduardo Utiyama ${ }^{2}$, Liliana Lotufo Oetting ${ }^{2}$, Pedro Agostinho Giani ${ }^{3}$, Urbano dos Santos Ruiz ${ }^{3}$, Valdomiro Shigueru Miyada ${ }^{4}$}

\author{
1 Pesquisa financiada pela FAPESP. \\ 2 Programa de Pós-graduação em Ciência Animal e Pastagens - Depto de Zootecnia, ESALQ/USP, CEP: 13418-900, Piracicaba/SP. \\ 3 Iniciação Cientifica, Dep. de Zootecnia da ESALQ/USP, Piracicaba/SP. \\ ${ }^{4}$ Depto de Zootecnia, ESALQ/USP, CEP 13418-900, Piracicaba/SP.
}

\begin{abstract}
RESUMO - Objetivou-se estudar os efeitos de probióticos, prebióticos e extratos vegetais como alternativas aos agentes antimicrobianos (promotores do crescimento), sobre a microbiota intestinal, a ocorrência de diarréia e o desempenho de leitões desmamados. Foram realizados dois experimentos em blocos casualizados, nos quais foram testados, durante 35 dias, cinco tratamentos: controle - ração basal; antimicrobiano - ração basal com 50 ppm de bacitracina de zinco + 50 ppm de olaquindox; probiótico - ração basal com 1.300 ppm de probiótico à base de Bacillus subtilis e Bacillus licheniformis; prebiótico - ração basal com 3.000 ppm de mananoligossacarídeo; extrato vegetal - basal com 500 ppm de extrato vegetal (alho, cravo, canela, pimenta, tomilho, cinamaldeído e eugenol). No Experimento 1, foram utilizados 40 leitões (idade inicial de 21 dias), alocados em 20 baias suspensas, cada uma com dois leitões (um macho castrado e uma fêmea - unidade) e quatro repetições por tratamento. No 350 dia do período experimental, um animal de cada unidade experimental foi abatido para coleta de amostras de conteúdo do jejuno/ íleo para análise microbiológica. No Experimento 2, foram utilizados 120 leitões, compondo 12 repetições por tratamento e dois animais (um macho castrado e uma fêmea) por unidade experimental. A freqüência de diarréia foi avaliada diariamente. Nenhum dos tratamentos foi eficaz em alterar a microbiota intestinal e a ocorrência de diarréia. Os agentes antimicrobianos melhoraram o ganho diário nos períodos de 15 a $35(+22 \%)$ e de 1 a $35(+21,4 \%)$ dias de experimentação. O probiótico e os extratos vegetais não promoveram benefício ao desempenho dos animais. O prebiótico, por sua vez, proporcionou desempenho equivalente ao tratamento antimicrobiano nos primeiros 14 dias experimentais, mas não melhorou a conversão alimentar.
\end{abstract}

Palavras-chave: desempenho, leitões recém-desmamados, microbiota intestinal, promotores de crescimento

\section{Effects of antimicrobials, prebiotics, probiotics and herbal extracts on intestinal microbiology, diarrhea incidence and performance of weanling pigs}

\begin{abstract}
The purpose of this work was to evaluate the effects of probiotics, prebiotics and herbal extracts as alternatives to antimicrobial agents (as growth promoters) on intestinal microbiology, diarrhea incidence, and on performance of weanling pigs. Two randomized complete block design experiments were carried out during 35 days to compare five treatments: control - basal diet; antimicrobial - basal diet plus Zn bacitracin and olaquindox ( $50 \mathrm{ppm}$ of each); probiotic - basal diet plus 1,300 ppm of probiotic (Bacillus subtilis and Bacillus licheniformis); prebiotic - basal diet plus 3,000 ppm of mannanoligosaccharide; herbal extract - basal diet plus $500 \mathrm{ppm}$ of herbal extract (garlic, clove, cinnamon, pepper, thyme, cinnamaldehyde, and eugenol). In the Experiment 1, forty 21 -d-weaned pigs were alloted to 20 suspended pens, each one with two pigs (one barrow and one gilt) per pen (experimental unit) and four replications per treatment. On $35^{\text {th }}$ day of experimental period, one animal of each experimental unit was slaughtered to collect the content of the jejunum/ileum for microbiological analysis. In the Experiment 2, one hundred and twenty 21-d-weaned pigs composed 12 replications per treatment and two animals (one barrow and one gilt) per experimental unit. Fecal score was daily evaluated to calculate diarrhea incidence. Treatment effect was observed neither on intestinal microbiology nor on diarrhea incidence. The antimicrobial agents improved average daily gain of weanling pigs, during 1-35 (+21.4\%) and 15-35 $(+22 \%)$ days of experimental periods, compared to pigs fed control diet. The probiotic and herbal extract did not improve piglet growth performance. The performance of piglets fed prebiotic was similar to those fed antimicrobials, during 1-14 days of experimental period. However, feed conversion was not improved.
\end{abstract}

Key Words: growth promoters, intestinal microbiota, performance, weanling pigs 


\section{Introdução}

Os antimicrobianos, quando utilizados como promotores do crescimento, possuem comprovada capacidade de aumentar o desempenho de suínos. Entretanto, seu uso tem sido restringido em diversos países, em virtude da possibilidade de desenvolvimento de resistência bacteriana cruzada (resulta em menor eficiência dos antimicrobianos na terapia animal e humana) e da emergente exigência dos importadores de produtos livres de resíduos de antibióticos (Silva, 2000).

Os probióticos, prebióticos e os extratos vegetais têm sido testados em dietas para suínos recém-desmamados como potenciais alternativas aos antibióticos e quimioterápicos como promotores do crescimento. Os probióticos podem atuar por diferentes mecanismos: exclusão competitiva e antagonismo direto (Menten, 2001), es tímulo ao sistema imune (Leedle, 2000), efeito nutricional (Leedle, 2000) e supressão da produção de amônia e neutralização de enterotoxinas (Jin et al., 1997). Os prebióticos, por sua vez, podem aderir a certos patógenos evitando sua adesão e colonização no epitélio intestinal (Macari \& Maiorka, 2000). Além disso, podem contribuir para a proliferação de microrganismos benéficos e estimular a resposta imunológica humoral (Ferket, 2002).

Por outro lado, os extratos vegetais ainda são pouco estudados. Entre os possíveis mecanismos de ação dos extratos vegetais no organismo animal, destacam-se o estímulo da digestão, as alterações na microbiota intestinal (efeito antimicrobiano) e a imunomodulação (Mellor, 2000).

Embora alguns efeitos tenham sido demonstrados, há ainda grande desconhecimento dos mecanismos envolvidos nos processos. Assim, este trabalho foi realizado com o objetivo de contribuir na elucidação dos efeitos da utilização de agentes antimicrobianos (bacitracina de zinco e olaquindox), probióticos (Bacillus subtilis e Bacillus licheniformis), prebióticos (mananoligossacarídeos) e extratos vegetais (alho, cravo, canela, pimenta, tomilho e os princípios ativos eugenol e cinamaldeído) sobre a microbiota intestinal, a freqüência de diarréia o desempenho de suínos recém-desmamados.

\section{Material e Métodos}

Foram conduzidos dois experimentos ( 35 dias cada um) em blocos casualizados na creche experimental do Setor de Suinocultura do Departamento de Zootecnia da Escola Superior de Agricultura "Luiz de Queiroz”, da Universidade de São Paulo. A creche experimental possuía baias metálicas suspensas, cada uma com área de $1,80 \mathrm{~m}^{2}$, providas de comedouros automáticos, bebedouro tipo chupeta e aquecimento complementar.

Foram avaliados cinco tratamentos: controle - ração basal; antimicrobiano - ração basal com 50 ppm de bacitracina de zinco e 50 ppm de olaquindox; probiótico ração basal com 1.300 ppm de probiótico à base de Bacillus subtilis e Bacillus licheniformis $\left(4,16 \times 10^{9}\right.$ de esporos viáveis por kg de ração); prebiótico - ração basal com 3.000 ppm de mananoligossacarídeo; extrato vegetal - ração basal com 500 ppm de extrato vegetal (alho, cravo, canela, pimenta, tomilho, cinamaldeído e eugenol).

Em ambos os experimentos, foram utilizadas duas dietas basais: pré-inicial (1으 ao 14 o dia de experimento) e inicial (15ㅇa ao 35o dia). Foram utilizados os valores preconizados pela Ajinomoto Biolatina Ind. e Com. Ltda. para determinação da composição dos alimentos e das exigências nutricionais. As composições percentuais das dietas basais, assim como os valores calculados de alguns nutrientes, são descritos na Tabela 1 .

No Experimento 1, para as análises microbiológicas, foram utilizados 40 leitões híbridos desmamados aos 21 dias de idade (peso inicial de $5,87 \pm 0,46 \mathrm{~kg}$ e peso final de $17,94 \pm 2,42 \mathrm{~kg}$ ), distribuídos em 20 baias (dois animais por baia), de acordo com o peso, totalizando quatro blocos (repetições) por tratamento. Os animais foram alimentados à vontade durante todo o período experimental (35 dias).

As análises microbiológicas foram realizadas em função do perfil de contaminação das instalações experimentais. Foram identificados Streptococcus spp., Staphylococcus spp., E.coli, Campylobacter coli e Clostridium perfringens em animais sadios e com diarréia, instalados na creche experimental antes do início dos experimentos.

No 35으 dia do experimento, um animal de cada unidade experimental foi abatido para coleta de amostras do conteúdo do jejuno/íleo (segmento de $10 \mathrm{~cm}$ retirado a $1 \mathrm{~m}$ da junção íleo-cecal) pelo método de raspagem. Após a coleta, as amostras foram submetidas às análises microbiológicas para identificação dos microrganismos totais, gram-positivos totais, gram-negativos totais, Bacillus spp., Streptococcus spp., Staphylococcus spp., Salmonella spp. e E. coli pelo método de plaqueamento preconizado por Lennette et al. (1985). A identificação e quantificação dos gêneros Campylobacter coli e Clostridium perfringens foram feitas de acordo com as técnicas descritas por Scarcelli et al. (1998) e Bressani (2001), respectivamente.

No Experimento 2, para determinação da freqüência de diarréia e análise do desempenho, foram utilizados 120 
Tabela 1 - Composição percentual e valores calculados de nutrientes das dietas pré-inicial ( 1 a 14 dias) e inicial (15 a 35 dias de experimento)

Table 1 - Percentage composition and calculated values of nutrients of pre-starter (1-14 days) and starter (15-35 days of experiment) basal diets

\begin{tabular}{|c|c|c|}
\hline $\begin{array}{l}\text { Ingrediente, \% } \\
\text { Ingredient }\end{array}$ & $\begin{array}{l}\text { Pré-inicial } \\
\text { Pre-starter }\end{array}$ & $\begin{array}{l}\text { Inicial } \\
\text { Starter }\end{array}$ \\
\hline Milho (Corn) & 53,80 & 61,72 \\
\hline Farelo de soja $46 \%$ (Soybean meal) & 19,00 & 25,00 \\
\hline Óleo de soja (Soybean oil) & 0,87 & 1,64 \\
\hline Calcário (Limestone) & 1,05 & 1,02 \\
\hline Fosfato bicálcico (Dicalcium phosphate) & 1,25 & 1,49 \\
\hline Soro de leite em pó ${ }^{1} 40 \%$ (Dried whey) & 10,50 & 2,30 \\
\hline Soro de leite em pó ${ }^{2} 70 \%$ (Dried whey) & 1,00 & 1,00 \\
\hline Lactose (Lactose) & 7,00 & 2,42 \\
\hline Plasma sangüíneo (Plasma) & 4,00 & 2,00 \\
\hline L-lisina. $\mathrm{HCl} 78 \%$ (L-lysine) & 0,47 & 0,38 \\
\hline L-treonina 98,5\% (L-threonine) & 0,18 & 0,14 \\
\hline L-triptofano $98 \%$ (L-tryptophan) & 0,06 & - \\
\hline DL-metionina $99 \%$ (DL-methionine) & 0,21 & 0,13 \\
\hline Sal (Salt) & - & 0,15 \\
\hline Antioxidante BHT (Antioxidant BHT) & 0,01 & 0,01 \\
\hline Cloreto de colina $60 \%$ (Choline chloride) & 0,10 & 0,10 \\
\hline Premix vitamínico(Vitamin premix $)^{3}$ & 0,10 & 0,10 \\
\hline Premix mineral (Mineral premix) ${ }^{4}$ & 0,10 & 0,10 \\
\hline Inerte (Inert) & 0,30 & 0,30 \\
\hline
\end{tabular}

Valor calculado, $\%$

Calculated value

\begin{tabular}{lrc}
\hline EM $(M E), \mathrm{kcal} / \mathrm{kg}$ & 3.400 & 3.300 \\
PB $(C P)$ & 20,00 & 19,50 \\
Lisina total (Total lysine) & 1,50 & 1,33 \\
Lisina digestível (Digestible lysine) & 1,34 & 1,19 \\
Treonina digestível (Digestible threonine) & 0,86 & 0,77 \\
Triptofano digestível (Digestible tryptophan) & 0,27 & 0,21 \\
Metionina digestível (Digestible methionine) & 0,46 & 0,39 \\
Met + cis digestíveis (Digestible met + cys) & 0,79 & 0,69 \\
Lactose (Lactose) & 11,76 & 4,00 \\
Ca & 0,85 & 0,87 \\
P disponível (Available P) & 0,45 & 0,42 \\
\hline
\end{tabular}

1 Soro de leite em pó com $40 \%$ de lactose (Dried whey - $40 \%$ lactose).

2 Soro de leite em pó com $70 \%$ de lactose (Dried whey - $70 \%$ lactose).

${ }^{3}$ Quantidades supridas por $\mathrm{kg}$ de ração (Supplemented amounts per $\mathrm{kg}$ of diet): vit. A, 6000 UI; vit. $D_{3}, 1500$ UI; vit. E, 15 UI; vit. $K_{3}, 1,5$ mg; tiamina (thiamin), $1,35 \mathrm{mg}$; riboflavina (riboflavin), $4 \mathrm{mg}$; vit. piridoxina(pyridoxine), $2 \mathrm{mg}$; vit. $\mathrm{B}_{12}, 20 \mathrm{mg}$; ácido nicotínico (nicotinic acid), $20 \mathrm{mg}$; ácido fólico (folic acid), 0,6 mg; biotina (biotin), 0,8 mg; ácido pantotênico (pantothenicacid), 9,35 mg; Se, 0,3 mg.

${ }^{4}$ Quantidades supridas por $\mathrm{kg}$ de ração (Supplemented amounts per kg of diet): I, 1,5 mg; Co, 1 mg; Cu, 10 mg; Zn 100 mg; Fe, 100 mg; Mn, 40 mg.

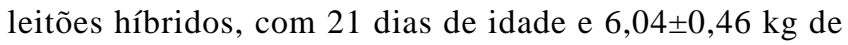
peso inicial, distribuídos em 60 baias (dois animais por baia) de acordo com o sexo e o peso, totalizando 12 blocos (repetições) por tratamento, sendo quatro blocos em cada um dos três períodos.

Os animais receberam água e ração à vontade. As variáveis de desempenho (consumo diário de ração, ganho diário de peso e conversão alimentar) foram calculadas por meio da pesagem e quantificação semanal das sobras de ração. A avaliação visual das fezes foi feita diariamente, atribuindo-se escores de 0 a 3 para cada animal: $0=$ fezes sólidas, $1=$ fezes pastosas, $2=$ fezes líquidas $/$ pastosas $\mathrm{e}$ 3 = fezes líquidas. Apenas os escores 2 e 3 indicavam a ocorrência de diarréia. Assim, pôde-se calcular a porcentagem de dias com ocorrência de diarréia nos períodos de 1 a 14,15 a 35 e 1 a 35 dias de experimento.

Os dados referentes à microbiologia intestinal, originalmente em ufc/g (unidades formadoras de colônias/g de amostra), foram transformados pela função $y=\log x$, em que $x$ é o número de unidades formadoras de colônias/g (ufc/g). Os dados de freqüência de diarréia, em \%, foram transformados pela função $y=\operatorname{arcsen} \sqrt{(p / 100)}$, de acordo com o recomendado por Barbin (2003), e posteriormente submetidos aos testes de adequação ao modelo linear, à análise de variâncias (PROC GLM do SAS 2001) e à comparação de médias pelo teste Tukey.

\section{Resultados e Discussão}

Os resultados obtidos nas análises microbiológicas (Experimento 1) são apresentados na Tabela 2. As variáveis Staphylococcus spp., Campylobacter coli, Clostridium perfringens e Salmonella spp. não foram analisadas. A presença de Staphylococcus (animal do tratamento extrato vegetal) e Campylobacter coli (animal do tratamento prebiótico) foi observada em apenas uma amostra, enquanto a de Clostridium perfringens foi identificada em quatro amostras (um animal de cada tratamento, exceto o tratamento probiótico). Os microrganismos Salmonela spp. não foram identificados em nenhuma das amostras. Nenhum dos tratamentos foi eficaz em definir um perfil específico da microbiota para as variáveis estudadas (microrganismos totais, gram-positivos totais, gram-negativos totais, Bacillus spp., Escherichia coli, Streptococcus spp., Staphylococcus spp., Campylobacter coli, Clostridium perfringens).

Como poucos animais apresentaram contaminação por esses microrganismos, não foi possível identificar o perfil de ação dos promotores de crescimento sobre parte da microbiota, possivelmente em decorrência de fatores como procedência e estado sanitário e nutricional dos animais experimentais, melhor qualidade das dietas experimentais e problemas na coleta e no transporte das amostras.

Os animais do Experimento 1 receberam na granja de origem, a partir do 70 dia de vida, uma alimentação complexa, livre de promotores de crescimento, e apresentavam excelentes condições sanitárias e corporais. Esses animais eram mais pesados e sadios que aqueles utilizados nas análises prévias de contaminação microbiológica. Esta diferença na 
Tabela 2 - Médias de microrganismos totais, gram-positivos totais, gram-negativos totais, Bacillus spp. e Escherichia coli no conteúdo intestinal do jejuno/íleo de leitões

Table 2 - Averages of total microorganisms, total gram positive microorganisms, total gram negative microorganisms, Bacillus spp. and Escherichia coli of jejunum/ileum contents of pigs

$\log _{10}$ ufc/g (unidades formadoras de colônia por grama de amostra)

$\log _{10} u f c / g$ (Units of colony/g sample)

\begin{tabular}{|c|c|c|c|c|c|c|}
\hline $\begin{array}{l}\text { Tratamento } \\
\text { Treatment }\end{array}$ & $\begin{array}{c}\text { Microrganismos } \\
\text { totais } \\
\text { Total microorganism }\end{array}$ & $\begin{array}{l}\text { Gram-positivas } \\
\text { totais } \\
\text { Total gram-positive }\end{array}$ & $\begin{array}{l}\text { Gram-negativas } \\
\text { totais } \\
\text { Total gram-negative }\end{array}$ & Bacillus spp. & Escherichia coli & Streptococcus spp. \\
\hline $\begin{array}{l}\text { Controle } \\
\text { Control }\end{array}$ & 5,511 & 5,416 & 4,994 & 3,861 & 4,383 & 3,794 \\
\hline Antimicrobiano & 5,857 & 5,659 & 5,033 & 4,838 & 3,969 & 3,000 \\
\hline $\begin{array}{l}\text { Antimicrobial } \\
\text { Probiótico } \\
\text { Probiotic }\end{array}$ & 6,245 & 6,184 & 4,865 & 4,966 & 4,032 & 4,364 \\
\hline $\begin{array}{l}\text { Extrato vegetal } \\
\text { Herbal extract }\end{array}$ & 5,957 & 5,766 & 4,810 & 4,882 & 3,622 & 4,442 \\
\hline $\begin{array}{l}\text { Média } \\
\text { Average }\end{array}$ & 5,866 & 5,705 & 4,886 & 4,309 & 3,923 & 4,138 \\
\hline $\begin{array}{l}\mathrm{Pr}>\mathrm{F} \\
\mathrm{CV}^{1}(\%)\end{array}$ & $\begin{array}{r}0,8671 \\
16,53\end{array}$ & $\begin{array}{r}0,8539 \\
18,34\end{array}$ & $\begin{array}{r}0,9808 \\
16,50\end{array}$ & $\begin{array}{r}0,1621 \\
28,35\end{array}$ & $\begin{array}{r}0,9185 \\
34,50\end{array}$ & $\begin{array}{r}0,4319 \\
37,40\end{array}$ \\
\hline
\end{tabular}

${ }^{1}$ Coeficiente de variação (Coefficient of variation).

saúde e no estado geral dos leitões pode talvez ser uma das causas da ausência de efeitos sobre a microbiota.

Os animais submetidos às análises prévias de microrganismos receberam uma dieta menos complexa, composta por milho, farelo de soja, pré-misturas mineral e vitamínica e óxido de zinco (preventivo de diarréias). Às dietas experimentais foram adicionados plasma sanguíneo spraydried, lactose, soro de leite em pó e aminoácidos sintéticos. Não foi adicionado nenhum promotor de crescimento nas dietas do tratamento controle. No entanto, as dietas continham 4 e $2 \%$ de plasma sangüíneo spray dried nas fases préiniciale inicial, respectivamente. Este ingrediente possui imunoglobulinas que auxiliam no equilíbrio da microbiota do leitão recém-desmamado, resultando em melhoras no desempenho e no estado de saúde (Kats et al., 1994; Gatnau et al., 1995; Caim \& Zimmerman, 1997). Assim, o plasma spray dried, aliado à qualidade de ingredientes lácteos, pode ter sido importante no controle da microbiota patogênica.

Além da procedência dos animais e da qualidade das dietas, deve-se considerar que problemas podem ter ocorrido durante a coleta e o transporte das amostras, como, por exemplo, a baixa sobrevivência das bactérias do momento da coleta à semeadura nos meios de cultivos. Segundo Apajalahti \& Bedford (1999), a coleta e o transporte das amostras são os pontos mais críticos na análise microbiológica em meios de cultura, pois uma exposição ao ar, mesmo que em períodos mínimos até o momento da semeadura, pode levar à alteração drástica no perfil e na quantidade de microrganismos. Por isso, as técnicas de cultivo em meios de cultura são limitadas e podem produzir dados muito pouco precisos ou equivocados (Apajalahti \& Bedford, 1999; Simpson et al., 1999). Segundo Apajalahti \& Bedford (1999), as técnicas moleculares de identificação e quantificação microbiana que estão sendo desenvolvidas são mais adequadas.

Outro argumento que pode acusar problemas nas análises microbiológicas está relacionado à ausência de efeito dos antimicrobianos sobre o perfil dos microrganismos analisados. Quando há desafio, ocorre melhora no desempenho dos animais alimentados com promotores de crescimento antimicrobianos. Este efeito está relacionado à ação dos antimicrobianos sobre a microbiota intestinal, pois, como demonstrado por Menten (1995), animais "germ-free" não são beneficiados.

Portanto, os possíveis problemas no processo de coleta, transporte e análise das amostras podem ter influenciado as análises microbiológicas, que, por sua vez, demonstraram não haver efeito dos promotores do crescimento sobre a microbiota.

A freqüência de diarréia não foi alterada $(\mathrm{P}>0,10)$ em nenhum dos períodos analisados. Portanto, nenhum dos tratamentos foi efetivo no controle de diarréias (Tabela 3).

Diversos fatores podem ter sido preponderantes na causa de diarréias pós-desmame, principalmente no período de 1 a 14 dias de experimentação. Segundo Kelly \& King (2001), o estresse da separação da porca, o transporte e o novo ambiente podem provocar alterações 
Tabela 3 - Médias de frequência de diarréia (MFD,\%) e média transformada (MT) para os períodos de 1 a 14,15 a 35 e 1 a 35 dias de experimentação

Table 3 - Average diarrhea frequency (MFD,\%) and transformed average (MT) from 1 to 14, 15 to 35 and 1 to 35 days of experimental periods

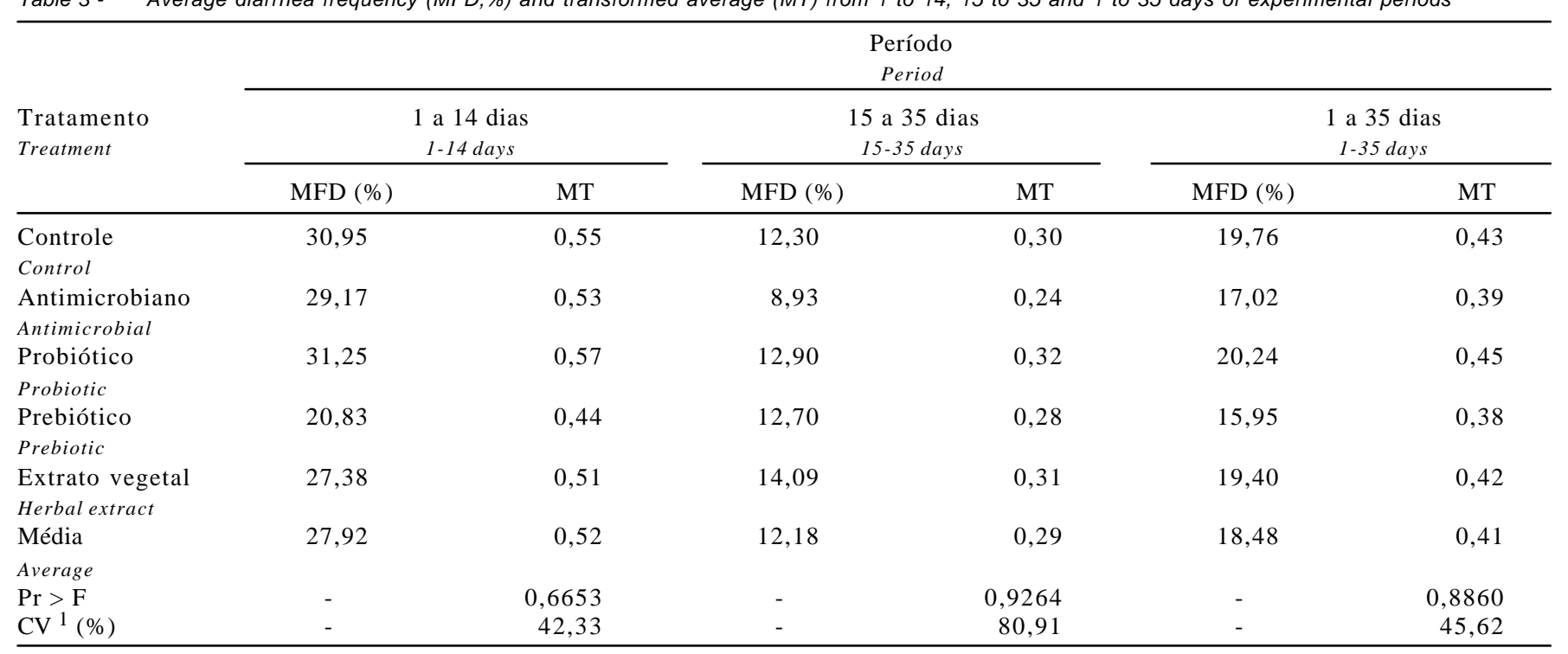

1 Coeficiente de variação (Coefficient of variation).

morfohistológicas importantes, deixando o leitão mais exposto às perturbações intestinais.

As diarréias no período pós-desmame também podem ser causadas pela colonização da superfície epitelial por patógenos, como a Escherichia coli enterotoxigênica, Salmonella typhimurium e Clostridium spp. (Stewart \& Chesson, 1993). Para fixação ao epitélio e colonização, as bactérias patógenas devem penetrar no muco glicoprotéico que reveste a superfície epitelial, por penetração quimiostática ou movimento ao longo de lacunas no muco (Vassalo et al., 1997). Além disso, resíduos alimentares não digeridos e não absorvidos servem como substratos para fermentação pela microbiota intestinal, elevando a produção de ácido lático e ácidos graxos voláteis. Estes substratos, juntamente com os resíduos alimentares ainda restantes e íons (sódio, potássio e cloreto), aumentam a osmolaridade do conteúdo intestinal. Assim, o processo de reabsorção de água é dificultado e ocorre um afluxo de água para a luz intestinal, desencadeando a diarréia (Etheridge et al., 1984; Nabuurs et al., 1993).

Portanto, outros fatores que fogem ao espectro de ação dos promotores do crescimento utilizados podem ter sido determinantes na ocorrência de diarréias.

As médias das variáveis de desempenho obtidas nos períodos de 1 a 14, 15 a 35 e 1 a 35 dias de experimentação são apresentadas nas Tabelas 4, 5 e 6, respectivamente.

Aos 14 dias de experimentação, o peso vivo (PV 14) e o ganho diário de peso dos animais do tratamento prebiótico foi superior $(\mathrm{P}<0,05)$ ao dos animais do tratamento extrato vegetal. Foi observado também maior $(\mathrm{P}<0,10)$ consumo diário de ração para os tratamentos antimicrobiano e prebiótico em relação ao tratamento extrato vegetal. Os tratamentos controle e probiótico, no entanto, não diferiram estatisticamente $(\mathrm{P}<0,10)$ de nenhum dos outros tratamentos e apresentaram valores intermediários de consumo. A conversão alimentar não foi afetada $(\mathrm{P}>0,10)$ pelos tratamentos.

No período de 15 a 35 dias de experimentação, o consumo diário de ração foi superior no tratamento antimicrobiano $(\mathrm{P}<0,05)$ em relação aos tratamentos probiótico e extrato vegetal. Os tratamentos prebiótico e controle não diferiram ( $\mathrm{P}>0,05)$ de nenhum outro tratamento. $\mathrm{O}$ peso vivo aos 35 dias de experimentação e o ganho diário de peso do tratamento antimicrobiano foram superiores $(\mathrm{P}<0,05)$ aos obtidos nos tratamentos controle, probiótico e extrato vegetal, que não diferiram $(\mathrm{P}>0,05)$. $\mathrm{O}$ tratamento prebiótico não diferiu $(\mathrm{P}>0,05)$ de nenhum outro e apresentou valor intermediário ao dos tratamentos controle e com antimicrobianos. A conversão alimentar não foi afetada ( $\mathrm{P}>0,05)$ pelos tratamentos.

No período de 1 a 35 dias de experimentação, o consumo diário de ração dos animais do tratamento antimicrobiano foi superior $(\mathrm{P}<0,05)$ ao obtido nos tratamentos extrato vegetal e probiótico. O consumo diário de ração no tratamento prebiótico não diferiu $(\mathrm{P}>0,05)$ dos obtidos nos tratamentos controle e antimicrobiano. Novamente, a conversão alimentar não foi afetada $(\mathrm{P}>0,05)$ pelos tratamentos.

O consumo diário de ração dos leitões do tratamento antimicrobiano foi superior ao dos animais do tratamento 
Tabela 4 - Médias de peso vivo (PV 14) aos 14 dias de experimentação, de consumo diário de ração (CDR 1-14), de ganho diário de peso (GDP 1-14) e conversão alimentar (CA 1-14) para o período de 1 a 14 dias de experimentação

Table 4 - Average body weight at 14 days (PV 14) of experiment, average daily feed intake (CDR 1-14), average daily weight gain (GDP 1-14) and feed conversion (CA 1-14) of weanling pigs from 1 to 14 days of experiment

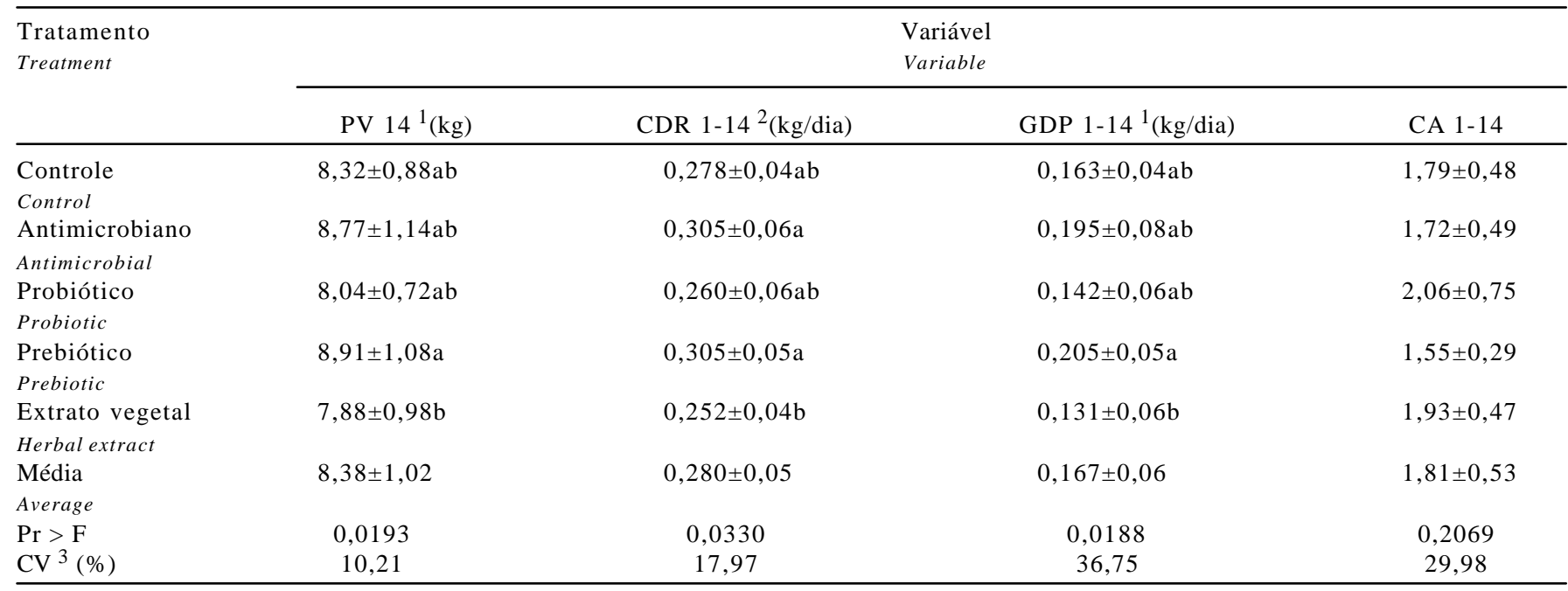

${ }^{1}$ Letras diferentes na coluna diferem $(P<0,05)$ significativamente pelo teste Tukey.

2 Letras diferentes, na coluna, diferem significativamente $(P<0,10)$ pelo teste Tukey.

3 Coeficiente de variação.

${ }^{1}$ Means with different subscript in each column differ $(P<0.05)$ by Tukey test.

${ }^{2}$ Means with different subscript in each column differ $(P<0.10)$ by Tukey test.

${ }^{3}$ Coefficient of variation.

Tabela 5 - Médias de peso vivo (PV 35) aos 35 dias de experimentação, consumo diário de ração (CDR 15-35), ganho diário de peso (GDP15-35) e conversão alimentar (CA 15-35) no período de 15 a 35 dias de experimentação

Table 5 - Average body weight at 35 days (PV 35) of experiment, average daily feed intake (CDR 15-35), average daily weight gain (GDP 15-35) and feed conversion (CA 15-35) of weanling pigs from 15 to 35 days of experiment

\begin{tabular}{|c|c|c|c|c|}
\hline \multirow[t]{2}{*}{$\begin{array}{l}\text { Tratamento } \\
\text { Treatment }\end{array}$} & \multicolumn{4}{|c|}{$\begin{array}{l}\text { Variável } \\
\text { Variable }\end{array}$} \\
\hline & PV $35^{1}(\mathrm{~kg})$ & CDR $15-35^{1}(\mathrm{~kg} / \mathrm{dia})$ & GDP $15-35^{1}(\mathrm{~kg} / \mathrm{dia})$ & CA $15-35$ \\
\hline $\begin{array}{l}\text { Controle } \\
\text { Control }\end{array}$ & $19,09 \pm 2,37 b$ & $0,817 \pm 0,16 \mathrm{ab}$ & $0,512 \pm 0,08 b$ & $1,59 \pm 0,15$ \\
\hline Antimicrobiano & $21,92 \pm 2,55 \mathrm{a}$ & $0,952 \pm 0,15 \mathrm{a}$ & $0,625 \pm 0,07 \mathrm{a}$ & $1,51 \pm 0,09$ \\
\hline $\begin{array}{l}\text { Antimicrobial } \\
\text { Probiótico } \\
\text { Probiotic }\end{array}$ & $18,50 \pm 2,71 b$ & $0,741 \pm 0,16 b$ & $0,498 \pm 0,10 b$ & $1,55 \pm 0,16$ \\
\hline $\begin{array}{l}\text { Prebiótico } \\
\text { Prebiotic }\end{array}$ & $20,84 \pm 2,97 \mathrm{ab}$ & $0,883 \pm 0,16 \mathrm{ab}$ & $0,568 \pm 0,10 \mathrm{ab}$ & $1,55 \pm 0,12$ \\
\hline Extrato vegetal & $18,47 \pm 2,53 b$ & $0,747 \pm 0,13 b$ & $0,503 \pm 0,08 b$ & $1,48 \pm 0,08$ \\
\hline $\begin{array}{l}\text { Herbal extract } \\
\text { Média } \\
\text { Average }\end{array}$ & $19,76 \pm 2,90$ & $0,828 \pm 0,17$ & $0,541 \pm 0,10$ & $1,53 \pm 0,13$ \\
\hline $\begin{array}{l}\mathrm{Pr}>\mathrm{F} \\
\mathrm{CV}^{2}(\%)\end{array}$ & $\begin{array}{l}0,0009 \\
11,34\end{array}$ & $\begin{array}{l}0,0004 \\
14,99\end{array}$ & $\begin{array}{l}0,0007 \\
14,47\end{array}$ & $\begin{array}{l}0,2336 \\
7,70\end{array}$ \\
\hline
\end{tabular}

${ }^{1}$ Letras diferentes na coluna diferem $(P<0,05)$ significativamente pelo teste Tukey.

2 Coeficiente de variação.

${ }^{1}$ Means with different subscript in each column differ $(P<0.05)$ by Tukey test.

${ }^{2}$ Coefficient of variation. 
Tabela 6 - Médias de consumo diário de ração (CDR 1-35), ganho diário de peso (GDP 1-35) e conversão alimentar (CA 1-35) no período de 1 a 35 dias de experimentação

Table 6 - Average daily feed intake (CDR 1-35), average daily weight gain (GDP 1-35) and feed conversion (CA 1-35) of weanling pigs from 1 to 35 days of experiment

\begin{tabular}{lccc}
\hline $\begin{array}{l}\text { Tratamento } \\
\text { Treatment }\end{array}$ & \multicolumn{3}{c}{$\begin{array}{c}\text { Variável } \\
\text { Variable }\end{array}$} \\
\cline { 2 - 4 } & $\begin{array}{c}\text { CDR 1-35 } \\
(\mathrm{kg} / \mathrm{dia})\end{array}$ & $\begin{array}{c}\text { GDP 1-35 } \\
(\mathrm{kg} / \mathrm{dia})\end{array}$ & CA 1-35 \\
\hline $\begin{array}{l}\text { Controle } \\
\text { Control }\end{array}$ & $0,602 \pm 0,11 \mathrm{abc}$ & $0,373 \pm 0,06 \mathrm{~b}$ & $1,61 \pm 0,12$ \\
$\begin{array}{l}\text { Antimicrobiano } \\
\text { Antimicrobial }\end{array}$ & $0,693 \pm 0,10 \mathrm{a}$ & $0,453 \pm 0,06 \mathrm{a}$ & $1,53 \pm 0,11$ \\
$\begin{array}{l}\text { Probiótico } \\
\begin{array}{l}\text { Probiotic } \\
\text { Prebiótico }\end{array}\end{array}$ & $0,549 \pm 0,11 \mathrm{c}$ & $0,356 \pm 0,08 \mathrm{~b}$ & $1,59 \pm 0,09$ \\
$\begin{array}{l}\text { Prebiotic } \\
\text { Extrato vegetal }\end{array}$ & $0,549 \pm 0,09 \mathrm{bc}$ & $0,354 \pm 0,07 \mathrm{~b}$ & $1,56 \pm 0,12$ \\
$\begin{array}{l}\text { Herbal extract } \\
\text { Média }\end{array}$ & $0,609 \pm 0,12$ & $0,392 \pm 0,08$ & $1,57 \pm 0,11$ \\
$\begin{array}{l}\text { Average } \\
\text { Pr }>\text { F }\end{array}$ & 0,0005 & 0,0009 & 0,2947 \\
CV 2 (\%) & 14,54 & 16,35 & 6,97 \\
\hline
\end{tabular}

${ }^{1}$ Letras diferentes na coluna diferem significativamente pelo teste Tukey $(\mathrm{P}<0,05)$.

2 Coeficiente de variação.

${ }^{1}$ Means with different subscript in each column differ $(P<0.05)$ by Tukey test. ${ }^{2}$ Coefficient of variation.

controle, proporcionando resultado superior no ganho diário de peso, principalmenteno período de 1 a 35 dias de experimentação, quando a melhora foi de $21,4 \%$ em comparação ao tratamento controle. O aumento no ganho diário de peso foi superior à média encontrada na literatura, de $11 \%$, calculada por Menten (1995) a partir de uma compilação de dados de experimentos realizados no Brasil. Esse resultado indica uma situação de elevado desafio aos leitões durante o período experimental, pois, quanto maior o desafio, maior o efeito dos antimicrobianos (Menten, 2001). Embora as análises microbiológicas não tenham identificado nenhuma alteração no perfil da microbiota de animais do tratamento antimicrobiano, é possível que tenha havido desafio microbiológico e que os antimicrobianos tenham agido sobre a microbiota, melhorando o desempenho dos leitões.

Ao contrário do tratamento antimicrobiano, o consumo diário de ração pelos animais do tratamento extrato vegetal, em todos os períodos, foi relativamente baixo, sendo numericamente inferior, inclusive, ao tratamento controle. Os extratos vegetais adicionados às dietas possuíam diversos fatores pungentes que podem ter causado esse efeito, pois, de acordo com a pesquisadora responsável pelo encapsulamento, o material encapsulante libera o conteúdo ao entrar em contato com a saliva, o que, possivelmente, foi uma das causas do baixo ganho de peso em todos os períodos.

Os extratos vegetais podem estimular a secreção de enzimas (amilase, sacarase e lipase), saliva, suco gástrico, suco pancreático e sais biliares (Sambaiah \& Srinivasan, 1991; Platel \& Srinivasan, 1996; Wang \& Bourne, 1998), mas esse tipo de efeito não refletiu na conversão alimentar dos leitões submetidos ao tratamento extrato vegetal, pois não houve diferença significativa em relação ao tratamento controle. No entanto, a diferença de $6,9 \%$ na conversão alimentar dos animais do tratamento extrato vegetal em relação ao tratamento controle no período de 15 a 35 dias de experimentação indica que esse efeito poderia ter sido identificado caso o número de repetições fosse mais elevado.

Situação semelhante ocorreu com o consumo diário de ração pelos leitões do tratamento probiótico, proporcionando ganho diário de peso inferior em todos os períodos. O resultado de desempenho do tratamento probiótico difere de algumas referências encontradas na literatura científica. Segundo Bellaver (2000), os resultados das pesquisas com probióticos serem muito variáveis, todavia, Stewart \& Chesson (1993) reuniram trabalhos na literatura utilizando diversos tipos de probióticos e concluíram que, em média, ocorre um aumento de 4,8\% no ganho diário de peso de leitões na fase inicial. Como o desempenho dos animais não foi melhorado, o probiótico utilizado pode não ter sido adequado à situação de desafio microbiano do experimento. Em alguns experimentos, o uso de probióticos à base de Bacillus toyoi (Vassalo et al., 1997) e Bacillus subtilis e Bacillus licheniformis (Kreuzer, 1994; Budiño, 2004) melhorou o ganho diário de peso de leitões recém-desmamados, porém, não havia desafio microbiológico nas instalações, pois não houve diferença entre os tratamentos controle e antimicrobiano. Esses resultados sugerem que o efeito do probiótico esteja relacionado ao grau de desafio das instalações utilizadas.

O resultado de ganho de peso do tratamento prebiótico, principalmente nos primeiros 14 dias de experimentação, foi ocasionado pelo maior consumo diário de ração, visto que a conversão alimentar não foi afetada pelo tratamento. Spring (2000) relatou alguns trabalhos científicos nos quais o uso de mananoligossacarídeo nas dietas de leitões recémdesmamados melhorou $(\mathrm{P}<0,05)$ o ganho diário de peso e o consumo diário de ração, mas não alterou a conversão alimentar. Este desempenho dos animais pode estar relacionado à ação do mananoligossacarídeo sobre a resposta imune. $\mathrm{O}$ mananoligossacarídeo tem demonstrado 
ser um agente promotor da imunidade humoral contra microrganismos patógenos específicos, evitando a colonização, permitindo sua apresentação às células do sistema imune (Ferket, 2003) e aumentando a presença de IgG no sangue e de IgA na bile e na mucosa intestinal (Savage et al., 1996). Com a ativação da resposta humoral, há economia de energia, pois a resposta inflamatória ativa despende de esforço energético maior.

\section{Conclusões}

O uso de antimicrobianos, de probióticos, de prebióticos e de extratos vegetais não alterou a microbiota intestinal e a frequência de diarréia em leitões recém-desmamados.

Os agentes antimicrobianos promoveram melhora no desempenho dos animais; o prebiótico proporcionou desempenho equivalente ao obtido com os antimicrobianos nos primeiros 14 dias de experimentação. O probiótico e os extratos vegetais, no entanto, não propiciaram nenhuma melhora nas características estudadas.

\section{Agradecimento}

À FAPESP, pelo financiamento do projeto de pesquisa.

À MCassab Com. e Ind. Ltda. e APC Inc. (plasma sangüíneo AP920 e lactose), à Ajinomoto Biolatina Ind. e Com. Ltda. (aminoácidos), à Sloten do Brasil Ind. e Com. Ltda. (soro de leite K-10 e K-21), à Alltech do Brasil Agroindustrial Ltda. (mananoligossacarídeo), à Givaudan do Brasil Ltda. (óleos essenciais e princípios ativos utilizados nos extratos vegetais), e à CHR Hansen Ind. e Com. Ltda. (probiótico Bio Plus 2B), pela doação de ingredientes para a realização dos experimentos.

À Professora Maria Inês Ré, responsável pelo Laboratório de Tecnologia de Partículas do IPT/USP, pela colaboração ao projeto no microencapsulamento dos óleos essenciais.

\section{Literatura Citada}

APAJALAHTI, J.; BEDFORD, M.R. Improve bird performance by feeding its microflora. World Poultry, v.15, n.2, p.20-23, 1999.

BARBIN, D. Planejamento e análise estatística de experimentos agropecuários. Arapongas: Midas, 2003. 194p.

BELLAVER, C. O uso de microingredientes (aditivos) na formulação de dietas para suínos e suas implicações na produção e na segurança alimentar. In: CONGRESSO MERCOSUL DE PRODUÇÃo SUÍNA, 2000, Buenos Aires. Anais... Buenos Aires: FCV, UBA; FAV, UNRC; EMBRAPA, 2000. p.93-108.

BRESSANI, M.A. Incidência de bactérias do grupo Bacteróides fragilis em crianças imunodeprimidas na cidade de São Paulo. São Paulo: Instituto de Ciências Biomédicas, 2001. 80p. Dissertação (Mestrado) - Instituto de Ciências Biomédicas/ Universidade de São Paulo, 2001.
BUDIÑO, F.E.L. Probiótico e/ou prebiótico na dieta de leitões recém-desmamados. Jaboticabal: Universidade Estadual Paulista, 2004. 75p. Tese (Doutorado em Zootecnia) Universidade Estadual Paulista, 2004.

CAIM, C.M.; ZIMMERMAN, D.R. Effect of spray dried plasma (SDP) on faecal shedding of hemolytic E. coli (HEC) and rotavirus by pigs in a segregated early-weaned (SEW) environment. Journal of Animal Science, v.75, p.61, 1997. (suppl. 1).

ETHERIDGE, R.D.; SEERLEY, R.W.; WYATT, R.D. The effect of the diet on performance, digestibility, blood composition and intestinal microflora of weaned pigs. Journal of Animal Science, v.58, n.6, p.1396-1402, 1984.

FERKET, P.R. Use of oligosaccharides and gut modifiers as replacements for dietary antibiotics. In: MINNESOTA NUTRITION CONFERENCE, 63., 2002, Minnesota. Proceedings... Minnesota: Eagan, 2002. p.169-182.

FERKET, P.R. Manutenção da saúde intestinal em um mundo sem antibióticos. In: RONDA LATINOAMERICANA DA ALLTECH, 13., 2003, Campinas. Anais... Campinas: ALLTECH, 2003. p.26-39.

GATNAU, R.; CAIN, C.; DREW, M. et al. Mode of action of spraydried porcine plasma in weanling pigs. Journal of Animal Science, v.73, p.82, 1995 (suppl. 1).

JIN, L.Z.; HO, Y.W.; ZHAO, X. Probiotics in poultry: modes of action. World's Poutry Science Journal, v.53, p.351-368, 1997.

KATS, L.J.; NELSSEN, J.L.; TOKACH, M.D. et al. The effect of spray-dried porcine plasma on growth performance in the earlyweaned pig. Journal of Animal Science, v.72, n.8, p.20752081, 1994.

KELLY, D.; KING, T.P. Digestive physiology and development in pigs. In: VARLEY, M.A.; WISEMAN, J. (Eds.). The weaner pig: nutrition and manegement. Nothingham: CABI Publishing, 2001. p.179-206.

KREUZER, M. Probiotic-antibiotic interactions in performance, intestinal fermentation and manure properties of piglets using a Bacillus (Bacillus subtilis e Bacillus licheniformis) preparation and carbadox. Agrobiological Research-zeitschrift fur Agrobiologie Agrikulturchemie Okologie. v.47, p.13-23, 1994 (abst.).

LEEDLE, J. Probiotics and DFM's - Mode of action in the gastrointestinal tract. In: SIMPÓSIO SOBRE ADITIVOS ALTERNATIVOS NA NUTRIÇÃO ANIMAL, Campinas, 2000. Anais... Campinas: Colégio Brasileiro de Nutrição Animal, 2000. p. 25-40

LENNETTE, J.K.; SPAUDING, L.H.; TRUANT, J.P. Manual of clinical microbiology. Washington: American Society for Microbiology, 1985. 314p.

MACARI, M.; MAIORKA, A. Função gastrintestinal e seu impacto no rendimento avícola. In: CONFERÊNCIA APINCO $2000 \mathrm{DE}$ CIÊNCIA E TECNOLOGIA AVÍCOLAS, Campinas, 2000. Anais... Campinas: Fundação Apinco de Ciência e Tecnologia Avícolas, 2000. p.1.61-174

MELLOR, S. Alternatives to antibiotic.Pig Progress, v.16, p.1821,2000 .

MENTEN, J.F.M. Eficácia, efeito sinérgico e modo de ação de agentes antimicrobianos como promotores do crescimento de suínos. Piracicaba: Escola Superior de Agricultura "Luiz de Queiroz", 1995. 106p. Tese (Livre Docência) - Escola Superior de Agricultura "Luiz".

MENTEN, J.F.M. Aditivos alternativos na produção de aves: probióticos e prebióticos. In: REUNIÃO ANUAL DA SOCIEDADE BRASILEIRA DE ZOOTECNIA, 38., 2001, Piracicaba. Anais... Piracicaba: Sociedade Brasileira de Zootecnia, 2001. p.141-157.

NABUURS, M.J.A; ZIJDERVELD, F.G.; DE LEEUW, P.W. Villus height and crypt depth in weaned and unweaned pigs, reared under various circumstances in the Netherlands. Research in Veterinary Science, v.55, p-78-84, 1993. 
PLATEL, K.; SRINIVASAN, K. Influence of dietary spices or their active principles on digestive enzymes of small intestinal mucosa in rats. International Journal of Food Sciences and Nutrition, v.47, p.55-59, 1996.

SAMBAIAH, K.; SRINIVASAN, K. Secretion and composition of bile in rats fed diets containing spices. Journal of Food Science and Technology, v.28, p.35-38, 1991.

STATISTICAL ANALYSIS SYSTEM - SAS. User's guide statistics. version 6.11. Cary: 1996. 634p.

SAVAGE, T.F.; COTTER, P.F.; ZAKREWSKA, E.I. The effect of feeding a mannan oligosaccharide on imunoglobulins, plasma IgG and bile IgA of Wrolstad MW male turkeys. Poultry Science, v.75, p.129S, 1996 (abst.) (suppl. 1).

SCARCELLI, E.; GENOVEZ, M.E.; CARDOSO, M.V. et al. Avaliação do potencial de disseminação de Campylobacter $\mathrm{spp}$ por diferentes espécies animais. Arquivos do Instituto Biológico, v.65, p.56-61, 1998.

SILVA, E.N. Antibióticos intestinais naturais: bacteriocinas. In: SIMPÓSIO SOBRE ADITIVOS ALTERNATIVOS NA NUTRIÇÃO ANIMAL, Campinas, 2000. Anais... Campinas: Colégio Brasileiro de Nutrição Animal, 2000. p.15-24.
SIMPSON, J.M.; McCRAKEN, V.J.; WHITE, B.A. et al. Application of denaturant gel electrophoresis for the analysis of the porcine gastrointestinal microbiota. Journal of Microbiology Methods, v.36, p.167-179, 1999.

SPRING, P. Yeast's secret weapon aids animal production. In: SIMPÓSIO SOBRE ADITIVOS ALTERNATIVOS NA NUTRIÇÃO ANIMAL, 2000, Campinas. Anais... Campinas: Colégio Brasileiro de Nutrição Animal, 2000. p.41-50.

STEWART, C.S.; CHESSON, A. Making sense of probiotics. Pig Veterinary Journal, v.31, p.11-33, 1993.

VASSALO, M.; FIALHO, E.T.; OLIVEIRA, A.I.G. et al. Probióticos para leitões dos 10 aos $30 \mathrm{~kg}$ de peso vivo. Revista Brasileira de Zootecnia, v.26, n.1, p.131-138, 1997.

WANG, R.; LI, D.; BOURNE, S. Can 2000 years of herbal medicine history help us solve problems in year 2000?. In: ALLTECH'S ANNUAL SYMPOSIUM, 14., 1998, Nottingham. Anais... Nottingham: ALLTECH, 1998. p.168-184.

Recebido: 30/08/04 Aprovado: 12/06/06 